\title{
Correction to: Class Aggregation for Multi-class Queueing Networks with FCFS Multi-server Stations
}

Pasquale Legato (D) and Rina Mary Mazza (D)

\author{
Correction to: \\ Chapter "Class Aggregation for Multi-class Queueing \\ Networks with FCFS Multi-server Stations" \\ in: T. Phung-Duc et al. (Eds.): Queueing Theory \\ and Network Applications, LNCS 11688, \\ https://doi.org/10.1007/978-3-030-27181-7_14
}

Unfortunately a few formulas were not displayed correctly in the contribution.

The correct formulas are given in this table:

\begin{tabular}{l|l|l}
\hline $\begin{array}{l}\text { Page \& } \\
\text { formula n }\end{array}$ & Errata & Corrige \\
\hline $\begin{array}{l}\text { pg. 3, } \\
\text { formula (5) }\end{array}$ & $\begin{array}{l}P_{j}(0 \mid \mathbf{n})= \\
1-1 / m_{j}\left[\sum_{c=1}^{c} R_{j} V_{j c} T_{c}(\mathbf{n})+\sum_{j=1}^{m_{j}-1}\left(m_{j}-l\right) P_{j}(l \mid \mathbf{n})\right]\end{array}$ & $\begin{array}{l}P_{j}(0 \mid \mathrm{n})= \\
1-1 / m_{j}\left[\sum_{c=1}^{c} R_{j} V_{j c} T_{c}(\mathbf{n})+\sum_{l=1}^{m_{j}-1}\left(m_{j}-l\right) P_{j}(l \mid \mathbf{n})\right]\end{array}$ \\
\hline pg. 8 & $\begin{array}{l}\text { Until convergence upon } \\
D_{j c}(\mathbf{N})=R_{j}, c=1, \ldots, C ; j=1, \ldots, M\end{array}$ & $\begin{array}{l}\text { Until convergence upon } \\
D_{j c}(\mathbf{N}), c=1, \ldots, C ; j=1, \ldots, M\end{array}$ \\
\hline $\begin{array}{l}\text { pg. 8, } \\
\text { formula (23) }\end{array}$ & $O\left(\left(M+\sum_{j=1}^{M} m_{j}\right) \cdot C\right)$ per iteration & $O\left(\sum_{j=1}^{M} m_{j} \cdot C\right)$ per iteration \\
\hline $\begin{array}{l}\text { pg. 10, } \\
\text { formula (26) }\end{array}$ & $O\left(\left(M+\sum_{j=1}^{M} m_{j}\right) \cdot(C+N)\right)$ per iteration & $O\left(\sum_{j=1}^{M} m_{j} \cdot(C+N)\right)$ per iteration \\
\hline pg. 11 & $\begin{array}{l}\text { Until convergence upon } \\
P_{j}(l-1 \mid N-1),\end{array}$ & $\begin{array}{l}\text { Until convergence upon } \\
P_{j}(l-1 \mid N-2) \text { and } P_{j}(l-1 \mid N-1), \\
l=1, \ldots, m_{j}-1 ; j=1, \ldots, M\end{array}$ \\
\hline
\end{tabular}

The updated version of this chapter can be found at https://doi.org/10.1007/978-3-030-27181-7_14 